\title{
The Beck Depression Inventory (BDI-II) and a single screening question as screening tools for depressive disorder in Dutch advanced cancer patients
}

\author{
Franca Warmenhoven • Eric van Rijswijk • \\ Yvonne Engels • Cornelis Kan • Judith Prins • \\ Chris van Weel $\cdot$ Kris Vissers
}

Received: 9 September 2010 /Accepted: 27 December 2010 / Published online: 18 January 2011

(C) The Author(s) 2011. This article is published with open access at Springerlink.com

\begin{abstract}
Purpose Depression is highly prevalent in advanced cancer patients, but the diagnosis of depressive disorder in patients with advanced cancer is difficult. Screening instruments could facilitate diagnosing depressive disorder in patients with advanced cancer. The aim of this study was to determine the validity of the Beck Depression Inventory (BDI-II) and a single screening question as screening tools for depressive disorder in advanced cancer patients.

Methods Patients with advanced metastatic disease, visiting the outpatient palliative care department, were asked to fill out a self-questionnaire containing the Beck Depression Inventory (BDI-II) and a single screening question "Are you feeling depressed?" The mood section of the PRIMEMD was used as a gold standard.
\end{abstract}

F. Warmenhoven $(\square) \cdot$ Y. Engels $\cdot$ K. Vissers

Department of Anesthesiology, Pain and Palliative Medicine,

Radboud University Nijmegen Medical Centre,

Huispost 630, P.O. Box 9101, 6500 HB, Nijmegen,

The Netherlands

e-mail: f.warmenhoven@anes.umcn.nl

E. van Rijswijk $\cdot$ C. van Weel

Department of Primary and Community Care,

Radboud University Nijmegen Medical Centre,

Nijmegen, The Netherlands

C. Kan

Department of Psychiatry,

Radboud University Nijmegen Medical Centre,

Nijmegen, The Netherlands

J. Prins

Department of Medical Psychology,

Radboud University Nijmegen Medical Centre,

Nijmegen, The Netherlands
Results Sixty-one patients with advanced metastatic disease were eligible to be included in the study. Complete data were obtained from 46 patients. The area under the curve of the receiver operating characteristics analysis of the BDI-II was 0.82 . The optimal cut-off point of the BDI-II was 16 with a sensitivity of $90 \%$ and a specificity of $69 \%$. The single screening question showed a sensitivity of $50 \%$ and a specificity of $94 \%$.

Conclusions The BDI-II seems an adequate screening tool for a depressive disorder in advanced cancer patients. The sensitivity of a single screening question is poor.

Keywords Depression - Advanced cancer V Validation .

Screening tool $\cdot$ BDI-II

\section{Introduction}

Depression seems highly prevalent in patients with advanced cancer. In different studies, a large variation of prevalence of depression in advanced cancer ( $4 \%$ to $58 \%$ ) is reported [1]. This large variation in prevalence can be explained by the use of different research samples with different risk factors like cancer type, age, sex, history of depression, alcohol abuses, and by the use of different assessment tools [2-4]. In some patients with advanced cancer, a depressive disorder as defined in the DSM-IV can be diagnosed, whereas others experience symptoms of depression and low mood but do not meet the diagnostic criteria of the DSM-IV. Both situations are associated with a lower quality of life and consequently cause a burden for the patient and his caregivers $[5,6]$.

In patients with advanced cancer, several factors can influence the diagnostic process of depression. Firstly, 
specific physical signs and symptoms presented in a patient with advanced cancer, such as weight loss or fatigue, can originate from advanced cancer or from depression [7]. Physical symptoms and depressive symptoms may partly share a common pathway of distress [8], which makes it difficult to distinguish between cause and effect: physical symptoms may increase a depressed feeling on the one hand and on the other hand a depressed feeling may result in more physical complaints $[9,10]$. Secondly, it may be difficult to distinguish grief from depression in patients with advanced cancer [11]. Thirdly, for both patient and physician emotional issues are difficult to address in this phase of life. Only a minority (17\%) of advanced cancer patients explicitly express their emotional distress to their physician and physicians themselves do not often address emotional problems in conversations with advanced cancer patients, possibly because they do not feel well trained in communication with palliative care patients $[12,13,17]$.

The recognition of depression is not optimal for oncologists [14, 15] and other physicians as well [16] because of the reasons mentioned. If patients with advanced cancer who are suffering from a depressive disorder are not identified, they will not be able to benefit from the pharmacological and psychological treatments that are considered beneficial $[16,18,19]$.

Screening instruments could facilitate recognizing depressive disorder in this population [20]. Systematic screening for symptoms like depression fits into the principles of advanced care planning that is promoted in patients with advanced cancer or in need of palliative care [21]. The ideal screening instrument combines a high sensitivity with a high specificity. When using a cut score in a screening instrument, the optimal cut score for a specific patient group can be determined with a receiver operating characteristics analysis. The usefulness of a screening tool depends on the positive and negative predictive value considering the main aim of the screening. When screening for depression in patients in a palliative trajectory, given the vulnerability of these patients and the consequences of depression for their health status and quality of life, a high negative predictive value seems most important. False positive cases can be ruled out in further diagnostic assessment.

Recently, screening for depression with one or two simple questions was suggested to be highly specific [22], although different studies report different findings on psychometric values of short screening methods [23]. A short screening method is appealing because the costs would be considerably lower than other methods for diagnosing depressive disorder; it is time efficient and many people can be screened for depression in a quick and simple way.

The Beck Depression inventory (BDI-II) is one of the most widely used screening tools for depression and it may provide a useful method for screening for depression in palliative care [24-26]. This inventory is a self-report questionnaire that was originally developed to rate the severity of depressive symptoms [27]. The BDI-II has shown good psychometric qualities as a screening tool for depression. However, the BDI-II contains several items on somatic symptoms of depression (for example, questions about loss of energy, fatigue, and loss of appetite), which may lead to an overestimation of positive cases in a patient group with somatic illness. The BDI-II has been validated in many samples including cancer patients [28].

The aim of the present study was to assess the validity of the BDI-II and a single screening question as screening tools for depression in Dutch-speaking patients with advanced cancer. The hypothesis was that both the BDI-II and the single screening question are adequate screening tools for depressive disorders in Dutch advanced cancer patients.

\section{Methods}

Ethical approval and informed consent

The study received approval of the medical ethical committee of the Radboud University Nijmegen Medical Centre. After obtaining informed consent, patients were asked to fill out a self-report questionnaire. Patients who were unable to read and understand the Dutch language were excluded from participating in this study.

\section{Sample and design}

From December 2003 until March 2007, all consecutive patients with advanced, non-curable stages of metastatic cancer referred to a new outpatient palliative care department at the Radboud University Nijmegen Medical Centre were asked to participate in the study by their treating physician. Patients considered by their physician to be too ill to participate were not asked.

\section{Questionnaires and data collection}

Patients were asked to provide demographic information. The BDI-II and a single screening question "Are you feeling depressed?" were used to screen for depression. The BDI-II is a 21-item self-report questionnaire with four response options for each item. Items of the BDI-II relate to different symptoms of depression such as sadness, hopelessness, self-blame, guilt, fatigue, and loss of appetite. On each item, patients are asked to choose the statement that best describes their attitude towards the item. Scores of the BDI-II can vary from 0 to 63 and are often classified as 
follows: 0-13 no depression, 14-19 mild depression, 20-28 moderate depression, and 29-63 severe depression [29].

Patients were asked to fill out the questionnaire during their visit at the outpatient department or at home. The mood section of the PRIME-MD [30] was administered as a gold standard for the clinical diagnosis of a depressive disorder by the physician, who was blind to the results of the questionnaire. The PRIME-MD is a structured interview based on the DSM-IV classification for depressive disorder and has been validated in oncology patients [31]. The PRIME-MD provides standardized questions that focus directly on key diagnostic symptoms and a depressive disorder was diagnosed when patients fulfilled the DSM-IV criteria. The time required to complete the PRIME-MD mood section is approximately $10 \mathrm{~min}$.

Statistical analysis

Statistical analysis was performed with SPSS 16.0. Means, sensitivity, specificity, and positive and negative predictive value were computed. A receiver operating characteristics (ROC) analysis was performed to determine the optimal cut score for the BDI-II as a screen for depression in this population.

\section{Results}

From December 2003 until March 2007, 61 patients (29 men, 32 women) were eligible to be included in the study. The inclusion period of this study has been relatively long because the outpatient department for palliative care patients was a new facility in the hospital and, consequently, referral numbers were low. Patients suffered from a variety of advanced cancers (23\% colon carcinoma, 16\% breast cancer, $8 \%$ head and neck cancer, $8 \%$ lung cancer, $45 \%$ some other malignancy). Of the 61 eligible patients, 20 patients $(33 \%)$ deceased within 6 months. Seven eligible patients refused to participate in the study.

Among the 54 patients who enrolled in the study, administration of the PRIME-MD was not completed in eight patients (Fig. 1). Therefore, complete data were obtained of 46 patients (26 women, 20 men, mean age 60 years, median age 58 years). The mean BDI-II score of the 46 patients who participated in the study was 14.7 (SD 9.9).

Ten out of 46 patients (22\%) were diagnosed with a major depressive disorder using the PRIME-MD. The area under the curve (AUC) of the ROC analysis of the BDI-II was 0.82 (Fig. 2). Using the traditional cut score of 14, the BDI-II demonstrated $90 \%$ sensitivity and $64 \%$ specificity. The positive and negative predictive values were $45 \%$ and $97 \%$, respectively. However, using a higher cut score of 16 retained high sensitivity $(90 \%)$ while increasing specificity to $69 \%$. The positive and negative predictive values were $55 \%$ and $96 \%$, respectively. The single screening question demonstrated $50 \%$ sensitivity and $94 \%$ specificity. The positive and negative predictive values of the single screening question were $71 \%$ and $87 \%$, respectively (Table 1).

\section{Discussion}

This study shows that the BDI-II is an adequate screening tool to detect depressive disorder in patients with advanced cancer. Using a cut-off point of 16 , it has good sensitivity (90\%) and acceptable specificity (69\%). The single screening question performed unsatisfactory, demonstrating only 50\% sensitivity and 94\% specificity. Given the consequences of not recognizing a depression

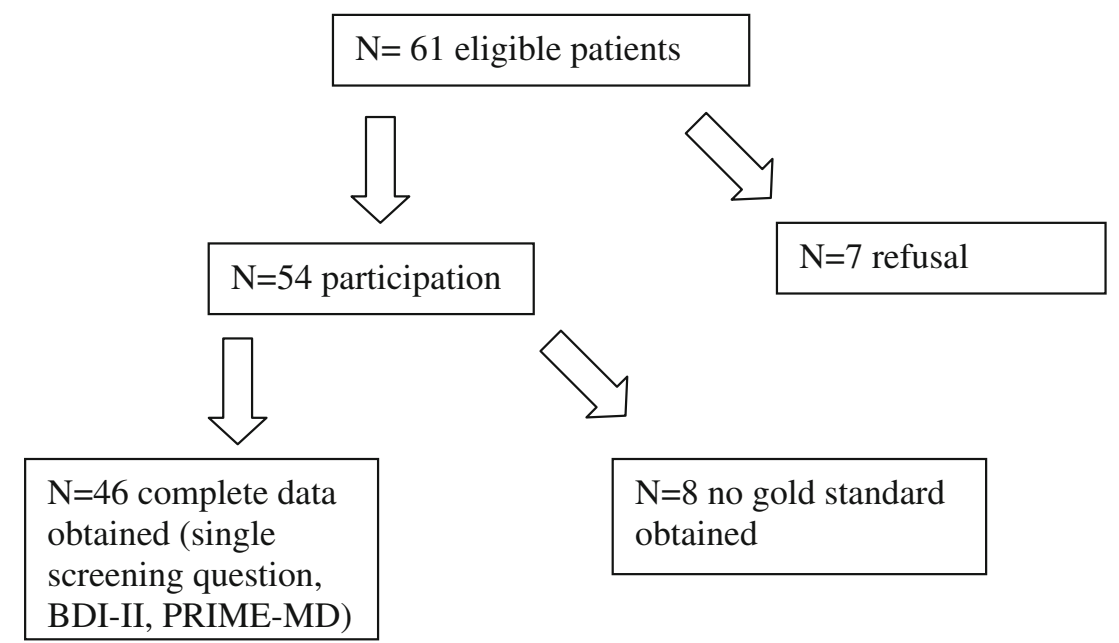

Fig. 1 Inclusion of patients 


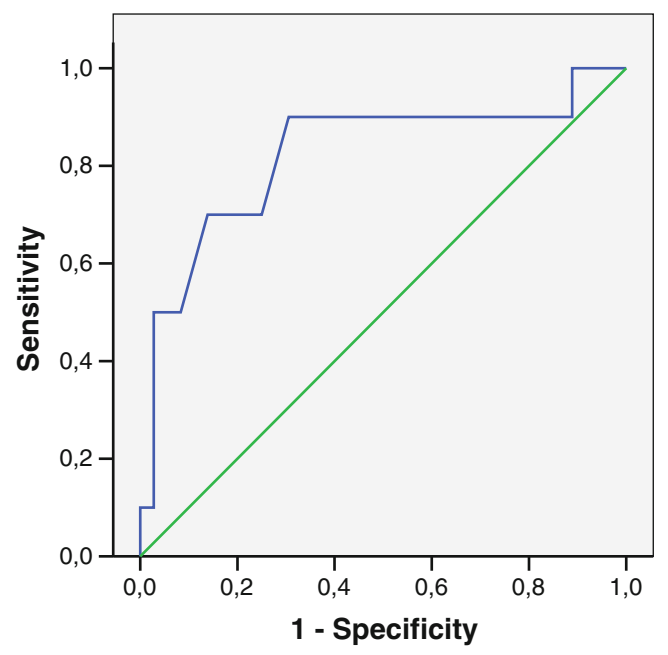

Fig. 2 Receiving operating characteristic beck depression inventory (BDI-II)

in this vulnerable patient group and the possibility to rule out depression in further diagnostic assessment in patients who screen positive for depression, a high sensitivity and high negative predictive value of screening tools are important and a lower specificity and positive predictive value are accepted.

The results of this study differ somewhat from previous findings on psychometric properties of the Beck Depression Inventory in a cancer patient sample. Katz et al. found that with a cut score of 16 the BDI had a sensitivity of $73 \%$ and specificity of $100 \%$ in a sample of 60 ambulatory patients with a malignancy of the head and neck region and identified the optimum cut score at 13 with a sensitivity of $92 \%$ and a specificity of $90 \%$ [32]. In the present study, the participants originated from a heterogeneous group of patients with advanced cancer, whereas Katz et al. used a more homogeneous group of ambulatory head and neck cancer patients. Furthermore, Katz et al. used a different instrument, the Schedule for Affective Disorders and Schizophrenia, as a gold standard.

A possible shortcoming of the BDI-II as a screening tool in advanced cancer patients is that the number of identified cases could be overestimated because of the somatic items of the questionnaire. The specificity of the BDI-II in advanced cancer patients might be increased by excluding the somatic items (e.g., weight loss, loss of appetite, etc.). The Beck Depression Inventory Short Form (BDI-SF), consisting of seven items tapping exclusively affective symptoms, might be more appropriate for this population. Love et al. performed a study in 227 women with metastatic breast disease in which the BDI-SF had a specificity of $63 \%$ and a positive predictive value of 0.52 [33]. This needs further study to determine the psychometric properties in a more heterogeneous patient group.

The sensitivity of the single screening question in this study appeared $50 \%$, which is almost similar to the reported sensitivity of $55 \%$ in an earlier study in 74 palliative care patients [34]. Other previous studies, however, report a much higher sensitivity of a single screening question in palliative care patients $(72-100 \%)[23,35]$. The low sensitivity of the single screening question in this study may point to difficulties regarding the meaning of the question. The word depression knows different translations and different interpretations in the Dutch language. The exact words that have been used might have influenced the results [36].

This study has some limitations. Firstly, the relatively small sample size was recruited from the recently started outpatient department for palliative care. Small sample size is not unusual in palliative care, in which recruitment is difficult and dropout rates are high due to deterioration of health status and death. Secondly, the selection of participants in this study was performed by the treating physician. The number of patients that the physicians considered too ill to participate was not recorded. In general, though, the participation of patients in research is high, when their "personal" treating physician invites them for participation, but it might introduce selection bias if the physician leaves out highly distressed patients. Given the relatively high scores on the BDI-II in this sample, severe bias seems unlikely. Thirdly, we used the PRIME-MD as a gold standard. The PRIME-MD and other psychiatric interviews are not validated for advanced cancer patients specifically. However, they have been validated in cancer patients [31].

This study offers a contribution to the diagnosis and screening of depressive disorder in advanced cancer patients. The Beck Depression Inventory offers a method for brief and sensitive detection of depressive disorder in this specific group of patients. If used systematically in

Table 1 Psychometric properties of single screening question and BDI-II

\begin{tabular}{lcccc}
\hline & Sensitivity (\%) & Specificity (\%) & Positive predictive value (\%) & Negative predictive value (\%) \\
\hline Single screening question & 50 & 69 & 71 & 87 \\
BDI-II (cut score 14) & 90 & 64 & 45 & 97 \\
BDI-II (cut score 16) & 90 & 69 & 55 & 96 \\
\hline
\end{tabular}


all advanced cancer patients, this screening tool may increase and improve physician's evaluations of not only somatic, but also psychological complaints of their patients.

To our knowledge, this is the first validation study of the Dutch Beck Depression Inventory and of a single screening question to screen for depression in a heterogeneous group of advanced cancer patients. Secondly, it gives a prevalence of depression in advanced cancer patients, based on a clinical diagnosis with the PRIME-MD, contrary to prevalence numbers acquired by questionnaires. In clinical practice, the screening tool can serve to alert physicians to the possibility of depressive disorder and hence give attention to the mental health status of palliative care patients, if necessary followed by further diagnostic assessment and appropriate treatment.

\section{Conclusions}

The BDI-II seems to be an adequate screening tool for depressive disorders in Dutch advanced cancer patients when using a cut score of 16 . The single screening question is less adequate to detect depression in palliative care patients, considering the low sensitivity.

Diagnosing depression in patients with advanced cancer remains a challenge. Using a valid screening tool can facilitate the process of diagnosing depression in this specific group of patients.

Conflict of interest The authors declare no conflict of interest relating to this manuscript. The authors have full control of the primary data and the data can be reviewed on request.

Open Access This article is distributed under the terms of the Creative Commons Attribution Noncommercial License which permits any noncommercial use, distribution, and reproduction in any medium, provided the original author(s) and source are credited.

\section{References}

1. Stiefel R, Die TM, Berney A, Olarte JM, Razavi A (2001) Depression in palliative care: a pragmatic report from the Expert Working Group of the European Association for Palliative Care. Support Care Cancer 9:477-488

2. Kelly J, Turner BJ (2009) Depression in advanced physical illness: diagnostic and treatment issues. Med J Aust 190:S90-S93

3. Massie MJ (2004) Prevalence of depression in patients with cancer. J Natl Cancer Inst Monogr (32):57-71

4. Reeve JL, Lloyd-Williams M, Dowrick C (2008) Revisiting depression in palliative care settings: the need to focus on clinical utility over validity. Palliat Med 22:383-391

5. Chochinov HM, Kristjanson LJ, Hack TF, Hassard T, McClement S, Harlos M (2007) Burden to others and the terminally ill. J Pain Symptom Manage 34:463-471
6. Henoch I, Bergman B, Gustafsson M, Gaston-Johansson F, Danielson E (2007) The impact of symptoms, coping capacity, and social support on quality of life experience over time in patients with lung cancer. J Pain Symptom Manage 34:370379

7. Hotopf M, Chidgey J, Ddington-Hall J, Ly KL (2002) Depression in advanced disease: a systematic review. Part 1. Prevalence and case finding. Palliat Med 16:81-97

8. Lo C, Zimmermann C, Rydall A, Walsh A, Jones JM, Moore MJ, Shepherd FA, Gagliese L, Rodin G (2010) Longitudinal study of depressive symptoms in patients with metastatic gastrointestinal and lung cancer. J Clin Oncol 28:3084-3089

9. Jacobsen PB, Donovan KA, Weitzner MA (2003) Distinguishing fatigue and depression in patients with cancer. Semin Clin Neuropsychiatry 8:229-240

10. Raison AH, Miller CL (2003) Depression in cancer: new developments regarding diagnosis and treatment. Biol Psychiatry 54:283-294

11. Periyakoil J, Hallenbeck VS (2002) Identifying and managing preparatory grief and depression at the end of life. Am Fam Physician 65:883-890

12. Anderson WG, Alexander SC, Rodriguez KL, Jeffreys AS, Olsen MK, Pollak KI, Tulsky JA, Arnold RM (2008) What concerns me is. Expression of emotion by advanced cancer patients during outpatient visits. Support Care Cancer 16:803811

13. Pollak KI, Arnold RM, Jeffreys AS, Alexander SC, Olsen MK, Abernethy AP, Sugg SC, Rodriguez KL, Tulsky JA (2007) Oncologist communication about emotion during visits with patients with advanced cancer. J Clin Oncol 25:57485752

14. Newell S, Sanson-Fisher RW, Girgis A, Bonaventura A (1998) How well do medical oncologists' perceptions reflect their patients' reported physical and psychosocial problems? Data from a survey of five oncologists. Cancer 83:1640-1651

15. Passik SD, Dugan W, McDonald MV, Rosenfeld B, Theobald DE, Edgerton S (1998) Oncologists' recognition of depression in their patients with cancer. J Clin Oncol 16:1594-1600

16. Lloyd-Williams M (2000) Difficulties in diagnosing and treating depression in the terminally ill cancer patient. Postgrad Med J 76:555-558

17. Barclay S, Wyatt P, Shore S, Finlay I, Grande G, Todd C (2003) Caring for the dying: how well prepared are general practitioners? A questionnaire study in Wales. Palliat Med 17:27-39

18. Akechi T, Okuyama T, Onishi J, Morita T, Furukawa TA (2008) Psychotherapy for depression among incurable cancer patients. Cochrane Database Syst Rev (2):CD005537

19. Hopko DR, Robertson SM, Carvalho JP (2009) Sudden gains in depressed cancer patients treated with behavioral activation therapy. Behav Ther 40:346-356

20. Kelly B, McClement S, Chochinov HM (2006) Measurement of psychological distress in palliative care. Palliat Med 20:779789

21. Murray SA, Sheikh A, Thomas K (2006) Advance care planning in primary care. BMJ 333:868-869

22. Arroll B, Goodyear-Smith F, Kerse N, Fishman T, Gunn J (2005) Effect of the addition of a "help" question to two screening questions on specificity for diagnosis of depression in general practice: diagnostic validity study. BMJ 331:884

23. Mitchell AJ, Kaar S, Coggan C, Herdman J (2008) Acceptability of common screening methods used to detect distress and related mood disorders-preferences of cancer specialists and nonspecialists. Psychooncology 17:226-236

24. Richter P, Werner J, Heerlein A, Kraus A, Sauer H (1998) On the validity of the Beck Depression Inventory. A review. Psychopathology 31:160-168 
25. Mystakidou K, Tsilika E, Parpa E, Smyrniotis V, Galanos A, Vlahos L (2007) Beck Depression Inventory: exploring its psychometric properties in a palliative care population of advanced cancer patients. Eur J Cancer Care Engl 16:244 250

26. Vodemaier A, Linden W, Siu C (2009) Screening for emotional distress in cancer patients: a systematic review of assessment instruments. J Natl Cancer Inst 101:1464-1488

27. Beck AT, Ward CH, Mendelson M, Mock J, Erbaugh J (1961) An inventory for measuring depression. Arch Gen Psychiatry 4:561571

28. Hopko DR, Bell JL, Armento ME, Robertson SM, Hunt MK, Wolf NJ, Mullane C (2008) The phenomenology and screening of clinical depression in cancer patients. J Psychosoc Oncol 26:3151

29. Beck AT, Steer RA, Brown GK (1996) Manual for the Beck Depression Inventory-II. Ref Type: Report

30. Fraguas R Jr, Henriques SG Jr, De Lucia MS, Iosifescu DV, Schwartz FH, Menezes PR, Gattaz WF, Martins MA (2006) The detection of depression in medical setting: a study with PRIMEMD. J Affect Disord 91:11-17
31. Leopold KA, Ahles TA, Walch S, Amdur RJ, Mott LA, WiegandPackard L, Oxman TE (1998) Prevalence of mood disorders and utility of the PRIME-MD in patients undergoing radiation therapy. Int J Radiat Oncol Biol Phys 42:1105-1112

32. Katz MR, Kopek N, Waldron J, Devins GM, Tomlinson G (2004) Screening for depression in head and neck cancer. Psychooncology 13:269-280

33. Love AW, Grabsch B, Clarke DM, Bloch S, Kissane DW (2004) Screening for depression in women with metastatic breast cancer: a comparison of the Beck Depression Inventory Short Form and the Hospital Anxiety and Depression Scale. Aust N Z J Psychiatry 38:526-531

34. Lloyd-Williams M, Dennis M, Taylor F (2004) A prospective study to compare three depression screening tools in patients who are terminally ill. Gen Hosp Psychiatry 26:384-389

35. Chochinov HM, Wilson KG, Enns M, Lander S (1997) "Are you depressed?" Screening for depression in the terminally ill. Am J Psychiatry 154:674-676

36. Noguera A, Centeno C, Carvajal A, Tejedor MA, Urdiroz J, Martinez M (2009) Spanish "fine tuning" of language to describe depression and anxiety. J Palliat Med 12:707-712 